\title{
QUALIDADE FISIOLÓGICA DE SEMENTES DE ALGODOEIRO TRATADAS COM REGULADORES DE CRESCIMENTO
}

\author{
Leonardo Cirilo da Silva Soares ${ }^{1}$, Rogério Lorençoni ${ }^{2}$, Adilson Nunes da Silva ${ }^{3}$, Juan Piero \\ Antonio Raphael ${ }^{4}$, Ederaldo José Chiavegato ${ }^{1}$, Durval Dourado Neto ${ }^{1}$ \\ ${ }^{1}$ Escola Superior de Agricultura Luiz de Queiroz - ESALQ/USP. Piracicaba, SP. E-mail: leonardo.cs.soares@gmail.com, \\ ${ }^{2}$ Universidade Federal do Tocantins, Campus de Gurupi. Gurupi, TO. E-mail: rogeriolorenconi@uft.edu.br \\ ${ }^{3}$ Universidade Federal da Bahia - UFBA. E-mail: nunesagr@gmail.com \\ ${ }^{4}$ Faculdade de Ciências Agronômicas, Campus de Botucatu - FCA/UNESP. Botucatu, SP. E-mail: juanpiero1@gmail.com
}

\section{RESUMO}

O objetivo deste trabalho foi avaliar o efeito de reguladores de crescimento, aplicados via tratamento de sementes, na qualidade fisiológica de sementes de algodão. Foram testados três reguladores de crescimento (cloreto de mepiquate; cloreto de mepiquate + ciclanilida; cloreto de clormequate) em cinco doses $(0 ; 1,5 ; 3,0 ; 4,5 ; 6,0 \mathrm{~g}$ de ingrediente ativo por $\mathrm{kg}$ de sementes). Realizou-se avaliações de condutividade elétrica, comprimento de radícula, comprimento de hipocótilo, comprimento total de plântulas, envelhecimento acelerado, germinação de sementes, e índice de velocidade de emergência de plântulas. Verificou-se que o tratamento de sementes com reguladores de crescimento interfere na qualidade fisiológica de sementes, reduzindo a germinação de sementes e a emergência de plântulas; e que há diferença entre os efeitos dos reguladores de crescimento.

Palavras-chave: Algodão, tratamento de sementes, cloreto de mepiquate

\section{PHYSIOLOGICAL QUALITY OF COTTON SEEDS TREATED WITH PLANT GROWTH REGULATORS}

\begin{abstract}
The objective of this study was to evaluate the effects of seed treatment with plant growth regulators on the physiological quality of cotton seeds. There were tested with three plant growth regulators (mepiquat chloride; mepiquat chloride+ cyclanilide; chlormequat chloride) at fivedoses $(0 ; 1.5 ; 3.0 ; 4.5 ; 6.0 \mathrm{~g}$ of active ingredient per $\mathrm{kg}$ of seeds $)$. Were realized evaluations of electrical conductivity, radicle length, hypocotyl length, seedling length, accelerated aging,
\end{abstract}


germination and emergence speed index. One conclude that seeds treated with plant growth regulators, by seed treatment, had an effect in the physiological quality of seeds, resulting in a decrease of germination and seedling emergence; there are different response from each plant growth regulators.

Keywords: Cotton, seed treatment, mepiquat chloride

\section{INTRODUÇÃO}

A utilização de reguladores de crescimento que proporcionam alterações na arquitetura de plantas vem sendo incrementada na cultura do algodoeiro há anos (CARVALHO et al., 1994; McCARTY e HEDIN, 1994). No Brasil, os produtos comerciais recomendados como reguladores de crescimento na cultura de algodoeiro, para redução da altura de plantas, têm mecanismos e modos de ação semelhantes, ou seja, interferem na biossíntese do ácido giberélico, inibindo-a, o que resulta em redução do crescimento e alterações fisiológicas (REDDY et al., 1995; MARUR, 1998).

Os reguladores de crescimento são substâncias químicas sintéticas que alteram o balanço hormonal das plantas, sendo que, em pequenas quantidades, promovem modificações nos processos fisiológicos, incluindo aumento ou diminuição das atividades metabólicas que resultam no crescimento, tais como alongamento $\mathrm{e}$ divisão celular, respiração, fotossíntese, síntese de proteínas, de lipídeos e de outras macromoléculas além de outros aspectos (CASTRO, 2006).

O momento em que é realizada a primeira aplicação na cultura é extremamente importante, pois havendo atraso, o efeito pode ser comprometido, sendo necessárias doses elevadas para que sejam obtidos resultados satisfatórios, e que podem não ser alcançados (LAMAS, 2001). Geralmente os reguladores de crescimento podem ser aplicados de maneira parcelada ao longo de todo o ciclo da cultura, ou através do monitoramento da altura das plantas (LACA-BUENDIA, 1989; LAMAS, 2001).

No caso da aplicação em função da altura da planta, independentemente do regulador utilizado, recomenda-se que a primeira aplicação seja realizada quando $50 \%$ das plantas apresentem os primeiros botões florais (estádio fenológico $\mathrm{B}_{1}$ de acordo com Marur e Ruano, 2001) e que apresentem alturas inferiores a $0,40 \mathrm{~m}$ (LAMAS, 2001). Entretanto, a grande 
maioria dos cultivares disponíveis no mercado apresenta crescimento bastante vigoroso e, dependendo das condições edafoclimáticas no referido estádio fenológico para a primeira aplicação, pode apresentar alturas superiores a $0,5 \mathrm{~m}$ (PAZZETTI et al., 2009), o que poderia levar a um controle ineficiente do crescimento das plantas. Portanto, em cultivos adensados, poderia ser utilizada a mesma estratégia adotada no controle das pragas e doenças iniciais do algodoeiro, ou seja, por meio do tratamento das sementes (NAGASHIMA et al., 2005).

De acordo com Taiz e Zeiger (2004), o processo de germinação de sementes de várias espécies exige a atuação de hormônios (giberelinas) para ativar o crescimento do embrião, o enfraquecimento da camada do endosperma que o envolve e restringe seu crescimento, e para a mobilização de reservas energéticas do endosperma. As giberelinas estão relacionadas com a síntese de enzimas hidrolíticas que degradam reservas, como o amido e as proteínas, que são usadas no desenvolvimento do embrião e também no alongamento da radícula. $\mathrm{Na}$ maioria das espécies, as giberelinas também atuam no alongamento celular, fazendo com que a raiz primária rompa os tecidos que restringem o seu crescimento, como o endosperma, o tegumento da semente ou estruturas do fruto (SALISBURY; ROSS, 1991; TAIZ; ZEIGER, 2004). Segundo Swain e Singh (2005), a inibição da síntese de giberelinas nas sementes pode afetar negativamente sua germinação.

Inúmeros trabalhos avaliaram o efeito de reguladores de crescimento aplicados via tratamento de sementes em plantas de algodão durante os estádios iniciais de desenvolvimento (NAGASHIMA et al., 2005; NAGASHIMA et al., 2010b; PAZZETTI et al., 2009; MARUR; RUANO, 2001; SOARES, 2010). No entanto, trabalhos que avaliem os efeitos do tratamento com reguladores de crescimento na qualidade fisiológica das sementes são escassos.

O objetivo deste trabalho foi avaliar os efeitos fisiológicos na qualidade de sementes de algodão tratadas com diferentes doses de reguladores de crescimento.

\section{MATERIAL E MÉTODOS}

O trabalho foi realizado em junho de 2010 no Laboratório de Tecnologia e Análise de Sementes da Universidade de São Paulo, da Escola Superior de Agricultura 
"Luiz de Queiroz" (ESALQ-USP), Piracicaba, SP.

O delineamento experimental utilizado foi o inteiramente casualizado, constituído por 14 tratamentos com 4 repetições. As sementes (cultivar FMT 523) foram submetidas a tratamentos com diferentes doses $(1,5 ; 3,0 ; 4,5$ e $6,0 \mathrm{~g}$ de ingrediente cloreto de mepiquate/clormequate por $\mathrm{kg}$ de semente) de três reguladores de crescimento [cloreto de mepiquate - CM (produto comercial PixHC); cloreto de mepiquate + ciclanilida $\mathrm{CM}+\mathrm{CY}$ (produto comercial Aplic); cloreto de clormequate - CC (produto comercial Tuval)]. Para comparação do efeito das doses foram utilizadas duas testemunhas (sem tratamento de sementes), sendo uma testemunha relativa embebida em água (mesmo volume dos demais tratamentos), e outra testemunha absoluta (sem embebição em água e sem tratamento de semente).

Durante o tratamento, as sementes foram embebidas em soluções preparadas com as respectivas doses do produto, completando-se com água destilada até obter-se um volume de $100 \mathrm{~mL} . \mathrm{kg}^{-1}$ de semente, sendo pré-hidratadas por três horas em temperatura ambiente antes da implantação dos testes fisiológicos: condutividade elétrica, crescimento de plântula, envelhecimento acelerado, germinação e, índice de velocidade de emergência.

\section{Condutividade elétrica (CE)}

Foi realizado com quatro subamostras de 50 sementes, que foram pesadas em balança de precisão e, posteriormente, colocadas em copos descartáveis de polietileno com volume de $300 \mathrm{~mL}$, contendo $75 \mathrm{~mL}$ de água destilada, os quais foram em seguida mantidos em incubadora BOD a $25^{\circ} \mathrm{C}$, por 24 horas. Após esse período, a condutividade elétrica da solução foi determinada por condutivímetro e os resultados foram expressos em $\mu \mathrm{S} \mathrm{cm}^{-1} \mathrm{~g}^{-1}$ de semente (VIEIRA; KRZYZANOWSKI, 1999). O valor obtido de cada amostra foi divido pelo respectivo peso das sementes.

\section{Crescimento de plântulas}

Foi utilizado o método de rolo de papel, umedecido com 2,5 vezes a massa do substrato. Na instalação do teste, as sementes foram depositadas sobre folhas de papel de germinação, distribuídas ao longo de um gabarito colocado no terço superior das folhas de papel, utilizando-se quatro repetições de 10 sementes, as quais foram 
colocadas com o hilo voltado para a parte inferior do papel, de maneira que durante o teste o crescimento do hipocótilo e da radícula não fosse comprometido. As avaliações foram realizadas cinco dias após a instalação do teste, determinando-se o comprimento da radícula (CR), do hipocótilo $(\mathrm{CH})$ e comprimento total das plântulas normais(CT).

\section{Germinação}

Foram utilizadas 200 sementes por tratamento, sendo 50 sementes por subamostra, colocadas para germinar em rolo de papel (tipo germitest) umedecido com 2,5 vezes a massa do substrato. Os rolos foram acondicionados em germinador a $25^{\circ} \mathrm{C}$, sendo as avaliações realizadas no quarto (G4) e sétimo (G7) dia após a instalação do teste, seguindo as Regras para Análise de Sementes (BRASIL, 1992). Os resultados foram expressos em porcentagem de plântulas normais.

\section{Envelhecimento acelerado}

Adotou-se metodologia descrita por Marcos Filho et al., (1987), com o uso de caixas plásticas $(11,5 \times 11,5 \times 3,5 \mathrm{~cm})$ como compartimentos individuais (mini-câmaras), possuindo em seu interior suportes para apoio de uma tela metálica, onde foram distribuídas as sementes em camada única. No interior de cada caixa foram adicionados $40 \mathrm{~mL}$ de água e as mesmas foram colocadas em câmara tipo BOD a $41^{\circ} \mathrm{C}$ por 48 h. Após o período de exposição, foi determinado o grau de umidade das sementes. Quatro subamostras de 50 sementes de cada tratamento foram colocadas para germinar, seguindo a mesma metodologia adotada na germinação. A avaliação foi realizada no quarto dia após a instalação do teste. Os resultados foram expressos em porcentagem de plântulas normais.

\section{Índice de velocidade de emergência (IVE)}

$\mathrm{O}$ teste foi conduzido com quatro repetições de 50 sementes para cada tratamento, semeadas em bandejas plásticas com as dimensões de $55 \mathrm{~cm}$ de comprimento, $35 \mathrm{~cm}$ de largura e $9 \mathrm{~cm} \mathrm{de}$ profundidade, sendo e distribuídas em sulcos de $20 \mathrm{~cm}$ de comprimento e $2 \mathrm{~cm}$ de profundidade, contendo como substrato areia lavada, umedecida com $60 \%$ da capacidade máxima de retenção de água (BRASIL, 1992). As bandejas foram mantidas em casa de vegetação, sendo o teste avaliado diariamente sempre no mesmo horário. 
Foram consideradas como plântulas normais todas aquelas emergidas com as folhas cotiledonares totalmente abertas e expandidas. Foi registrado diariamente o número de plântulas normais no período de 16 dias após a semeadura (DAS) para obter o índice de velocidade de emergência (IVE). Para o cálculo do IVE, utilizou-se a fórmula de Maguire (1962), na qual IVE = N1/D1 + $\mathrm{N} 2 / \mathrm{D} 2+\ldots+\mathrm{Nn} / \mathrm{Dn}$, onde: IVE $=$ índice de velocidade de emergência de plântulas; $N=$ número de plântulas emergidas e computadas da primeira à última contagem; $\mathrm{D}=$ número de dias da semeadura da primeira a última contagem.

Os resultados obtidos foram submetidos às seguintes análises estatísticas: (i) teste t de Student (dados não pareados) para comparação das médias das testemunhas (com e sem embebição em água); (ii) análise de variância para verificar a existência de diferenças significativas entre médias de produtos e testemunha, tendo sido anteriormente realizados o teste de Hartley (1950) para verificar a homogeneidade das variâncias e a transformação de dados por meio da família de transformação ótima de Box-Cox (1964); (iii) teste Tukey, ao nível de $5 \%$ de probabilidade, para comparação de médias entre produtos e testemunha; (iv) regressão linear simples, para verificar efeito de dose dos produtos testados. As análises foram realizadas utilizando pacote estatístico do programa SAS, versão 9.2 (SAS Institute Inc., Cary, NC, USA).

\section{RESULTADOS E DISCUSSÃO}

De acordo com os resultados da comparação de sementes (testemunhas) não embebidas e embebidas em água verifica-se diferença apenas para determinação de condutividade elétrica (Tabela 1). Porém, para os demais atributos avaliados (crescimento de plântula, envelhecimento acelerado, germinação e, índice de velocidade de emergência) não houve diferença dos resultados de sementes embebidas e não embebidas em água. Dutra e Medeiros Filho (2009), avaliando condutividade elétrica de sementes de algodão herbáceo sob diferentes tratamentos de pré-hidratação, verificaram uma alta relação entre a condutividade elétrica conduzida nas sementes e o tratamento sem pré-hidratação. Portanto, foram utilizados no presente trabalho os valores da testemunha relativa (embebida em água) para comparação com os tratamentos que receberam diferentes doses de reguladores de crescimento, desta forma todos os tratamentos analisados receberam o mesmo volume de água. 
Foram detectadas diferenças significativas entre os tratamentos para todos os parâmetros analisados (Tabela 2). Os resultados evidenciaram que, para o tratamento CM, houve redução de 19\% no valor de G4 (Tabela 3). O tratamento $\mathrm{CM}+\mathrm{CY}$ aumentou o valor de CE em $15 \%$ e diminuiu os valores de $\mathrm{CH}$, EA, IVE, G4 E
G7 em $30 \%, 47 \%, 49 \%, \quad 17 \%$ e $15 \%$, respectivamente (Tabela 3 ). $\mathrm{O}$ tratamento $\mathrm{CC}$ reduziu os valores de $\mathrm{CH}, \mathrm{CR}, \mathrm{CT}, \mathrm{G} 4 \mathrm{e}$ G7 em 27\%, 35\%, 32\%, 31\% e 29\%, respectivamente (Tabela 3). Não houve diferença significativa entre tratamentos que diferiram da testemunha.

Tabela 1. Médias e p-valor (teste $\mathrm{t}$ de Student) para comparação dos resultados obtidos em função dois tratamentos. Condutividade Elétrica (CE, $\mu \mathrm{S} \mathrm{cm} \mathrm{c}^{-1} \mathrm{~g}^{-1}$ de semente), Comprimento de Raiz (CR, cm), Comprimento de Hipocótilo ( $\mathrm{CH}, \mathrm{cm})$, Comprimento total (CT, cm), Envelhecimento Acelerado - germinação (EA, \%), Germinação aos quatro dias (G4, \%), Germinação aos sete dias (G7, \%) e, Índice de velocidade de emergência (IVE), Piracicaba (SP), 2010.

\begin{tabular}{ccccccccc}
\hline Tratamento & CE & CH & CR & CT & EA & G4 & G7 & IVE \\
\hline Testemunha (sem água) & 1491,3 & 2,57 & 5,13 & 7,70 & 34,5 & 82,0 & 84,0 & 3,11 \\
Testemunha (com água) & 1163,5 & 2,93 & 5,55 & 8,48 & 52,0 & 86,5 & 87,5 & 2,69 \\
\hline$p-$ valor $^{l}$ & $0,0148^{*}$ & $0,1551^{\mathrm{ns}}$ & $0,2927^{\mathrm{ns}}$ & $0,1658^{\mathrm{ns}}$ & $0,0886^{\mathrm{ns}}$ & $0,5356^{\mathrm{ns}}$ & $0,6040^{\mathrm{ns}}$ & $0,2279^{\mathrm{ns}}$ \\
\hline
\end{tabular}

${ }^{1}$ Teste $t$ de Student (não pareado). *Médias diferem (na coluna) ao nível de pelo menos 5\% de probabilidade. ns - não significativo, médias não diferem.

Tabela 2. Resumo da Análise de Variância (p-valor e coeficiente de variação - CV) para comparação entre tratamentos (testemunha embebida em água e médias de doses de cada regulador de crescimento). Condutividade Elétrica (CE, $\mu \mathrm{S} \mathrm{cm-1} \mathrm{g-1} \mathrm{de}$ semente), Comprimento de Raiz ( $\mathrm{CR}, \mathrm{cm})$, Comprimento de Hipocótilo $(\mathrm{CH}, \mathrm{cm})$, Comprimento total $(\mathrm{CT}, \mathrm{cm})$, Envelhecimento Acelerado - germinação (EA, \%), Germinação aos quatro dias (G4, \%), Germinação aos sete dias (G7, \%) e, Índice de velocidade de emergência (IVE). Piracicaba (SP), 2010.

\begin{tabular}{cccccccccc}
\hline $\begin{array}{c}\text { Causa de } \\
\text { variação }\end{array}$ & $\mathrm{G}$ & $\mathrm{CE}$ & $\mathrm{CH}$ & $\mathrm{CR}$ & $\mathrm{CT}$ & $\mathrm{EA}$ & $\mathrm{G} 4$ & $\mathrm{G} 7$ & $\mathrm{IVE}$ \\
\hline Tratamento & 3 & $\begin{array}{c}0.0072 \\
*\end{array}$ & $\begin{array}{c}0.0441 \\
*\end{array}$ & $\begin{array}{c}0,0069 \\
*\end{array}$ & $\begin{array}{c}0,034 \\
*\end{array}$ & $\begin{array}{c}<0,0001 \\
*\end{array}$ & $\begin{array}{c}0,0006 \\
*\end{array}$ & $\begin{array}{c}0,0001 \\
*\end{array}$ & $\begin{array}{c}<0,0001 \\
*\end{array}$ \\
Resíduo & 48 &.. &.. &.. &.. &.. &.. &.. &.. \\
Total & 51 &.. &.. &.. &.. &.. &.. &.. &.. \\
\hline $\mathrm{CV}(\%)$ &.. & 11,5 & 26,23 & 29,86 & 27,45 & 25,27 & 16,92 & 14,26 & 23,96 \\
\hline
\end{tabular}

1 p-valor:Teste F. *Pelo menos duas médias diferem ao nível 5\% de probabilidade. ns - não significativo, médias não diferem. 
Verificou-se que todos os parâmetros avaliados, exceto CR, sofreram efeito de dose no tratamento CM +CY (Tabela 4). Para o tratamento $\mathrm{CC}$, houve efeito de dose para os parâmetros $\mathrm{CH}, \mathrm{CR}, \mathrm{CT}$ e EA (Tabela 4). Efeito de dose para o tratamento $\mathrm{CM}$ foi verificado somente para $\mathrm{CH}$ e EA (Tabela 4).Verificou-se que o produto $\mathrm{CM}+\mathrm{CY}$ promoveu maior condutividade elétrica, o que corresponde a uma provável redução do vigor em relação à testemunha (Tabela 3), e também foi o único produto que promoveu efeito de dose (Tabela 4), sendo que a cada grama do ingrediente ativo a condutividade elétrica aumentou em 5,8\% $\left(64,78 \mu \mathrm{S} \mathrm{cm}^{-1} \mathrm{~g}^{-1}\right.$ de semente). Os produtos CM e CC não diferiram da testemunha, e não houve diferença entre os reguladores de crescimento (Tabela 3).

Observando os resultados de comprimento de plântula verificou-se que o produto $\mathrm{CC}$ foi efetivo na redução do crescimento, pois diferiu da testemunha nas avaliações de comprimento de raiz, comprimento de hipocótilo e comprimento total (Tabela 3). O produto $\mathrm{CM}+\mathrm{CY}$ reduziu o comprimento de hipocótilo, sem afetar raiz e comprimento total. Já as médias do produto CM não diferiram da testemunha em nenhuma das determinações de comprimento de plântula. Porém, não houve diferença entre os reguladores de crescimento.

Soares (2010) verificou que os reguladores de crescimento cloreto de mepiquate e cloreto de mepiquate associado à ciclanilida interferem no crescimento de raízes secundárias, sendo que o efeito da associação à ciclanilida promove maior redução do crescimento radicular.

Todos os reguladores promoveram efeito de dose (Tabela 4) para comprimento de hipocótilo, sendo que a cada grama de ingrediente ativo por $\mathrm{kg}$ de semente houve redução no crescimento de $8,4 \%$ para $\mathrm{CC}$, 7,0\% para $\mathrm{CM}+\mathrm{CY}$ e, de $4,2 \%$ para $\mathrm{CM}$. Esses resultados estão de acordo com dados obtidos por Nagashima et al. (2007, 2010a), Lamas (2006) e Soares (2010), que verificaram que o uso de reguladores de crescimento via tratamento de sementes reduz o crescimento inicial em altura da parte aérea de plantas de algodão.

Para comprimento de raiz, $\mathrm{CC}$ foi o único produto que promoveu significativa redução do crescimento em função do aumento de dose. A cada grama de ingrediente ativo por $\mathrm{kg}$ de semente aplicado houve redução de 9,1\% (Tabela 4). 
Tabela 3. Comparação de médias entre tratamentos (testemunha embebida em água e médias de doses de cada regulador de crescimento). Condutividade Elétrica (CE, $\mu \mathrm{S} \mathrm{cm} \mathrm{g}^{-1} \mathrm{de}$ semente), Comprimento de Raiz (CR, cm), Comprimento de Hipocótilo $(\mathrm{CH}, \mathrm{cm})$, Comprimento total $(\mathrm{CT}, \mathrm{cm})$, Envelhecimento Acelerado - germinação (EA, \%), Germinação aos quatro dias (G4, \%), Germinação aos sete dias (G7, \%) e, Índice de velocidade de emergência (IVE), Piracicaba (SP), 2010.

\begin{tabular}{lllllllll}
\hline Tratamento & CE & CH & CR & CT & EA & G4 & G7 & IVE \\
\hline Testemunha & $1163,50 \mathrm{~b}$ & $2,93 \mathrm{a}$ & $5,55 \mathrm{a}$ & $8,48 \mathrm{a}$ & $52,00 \mathrm{a}$ & $86,50 \mathrm{a}$ & $87,50 \mathrm{a}$ & $2,69 \mathrm{a}$ \\
$\mathrm{CM}+\mathrm{CY}$ & $1343,38 \mathrm{a}$ & $2,03 \mathrm{~b}$ & $5,23 \mathrm{ab}$ & $7,26 \mathrm{ab}$ & $27,38 \mathrm{~b}$ & $72,00 \mathrm{~b}$ & $74,12 \mathrm{bc}$ & $1,36 \mathrm{~b}$ \\
$\mathrm{CM}$ & $1223,06 \mathrm{ab}$ & $2,34 \mathrm{ab}$ & $4,57 \mathrm{ab}$ & $6,91 \mathrm{ab}$ & $42,38 \mathrm{a}$ & $69,75 \mathrm{~b}$ & $77,50 \mathrm{ab}$ & $2,38 \mathrm{a}$ \\
$\mathrm{CC}$ & $1171,13 \mathrm{ab}$ & $2,13 \mathrm{~b}$ & $3,62 \mathrm{~b}$ & $5,75 \mathrm{~b}$ & $55,75 \mathrm{a}$ & $59,30 \mathrm{~b}$ & $63,25 \mathrm{c}$ & $2,73 \mathrm{a}$
\end{tabular}

Médias seguidas por letras iguais na coluna, não diferem pelo teste Tukey ao nível de 5\% de probabilidade.

Tabela 4. Análise de regressão para as diferentes doses dos reguladores de crescimento CM, $\mathrm{CM}+\mathrm{CY}$ e CC. Condutividade Elétrica (CE, $\mu \mathrm{S} \mathrm{cm}^{-1} \mathrm{~g}^{-1}$ de semente), Comprimento de Raiz (CR, cm), Comprimento de Hipocótilo $(\mathrm{CH}, \mathrm{cm})$, Comprimento total (CT, $\mathrm{cm}$ ), Envelhecimento Acelerado - germinação (EA, \%), Germinação aos quatro dias (G4, \%), Germinação aos sete dias $(\mathrm{G} 7, \%)$ e, Índice de velocidade de emergência (IVE). Piracicaba (SP), 2010.

\begin{tabular}{|c|c|c|c|c|c|c|c|c|c|c|}
\hline \multirow[b]{2}{*}{$\mathrm{P}$} & \multicolumn{4}{|c|}{$\mathrm{CM}$} & \multicolumn{3}{|c|}{$\mathrm{CM}+\mathrm{CY}$} & \multicolumn{3}{|c|}{$\mathrm{CC}$} \\
\hline & Equação & & $\mathrm{R}^{2}$ & $\begin{array}{c}p- \\
\text { valor }\end{array}$ & Equação & $\mathrm{R}^{2}$ & $\begin{array}{c}p- \\
\text { valor }\end{array}$ & Equação & $\mathrm{R}^{2}$ & $\begin{array}{c}p- \\
\text { valor }\end{array}$ \\
\hline $\mathrm{CE}$ & $y=20,500 x+115$ & & $\begin{array}{c}0,092 \\
1\end{array}$ & 0,1934 & $y=64,783 x+111$ & $\begin{array}{c}0,684 \\
1\end{array}$ & $<0,0001$ & $y=21,083 x+1170$ & $\begin{array}{c}0,170 \\
9\end{array}$ & 0,0701 \\
\hline $\mathrm{CH}$ & $\begin{array}{l}y=-0,118 x \\
2,811\end{array}$ & + & $\begin{array}{c}0,216 \\
9\end{array}$ & 0,0385 & $\begin{array}{l}y=-0,196 x \\
2,799\end{array}$ & $\begin{array}{c}0,595 \\
4\end{array}$ & $<0,0001$ & $y=-0,2583 x+3,064$ & $\begin{array}{c}0,523 \\
4\end{array}$ & 0,0003 \\
\hline $\mathrm{CR}$ & $\begin{array}{l}y=-0,195 x \\
5,353\end{array}$ & + & $\begin{array}{c}0,116 \\
3\end{array}$ & 0,1412 & $\begin{array}{l}y=-0,126 x \\
5,674\end{array}$ & $\begin{array}{c}0,062 \\
3\end{array}$ & 0,2886 & $y=-0,4985 x+5,501$ & $\begin{array}{c}0,426 \\
0\end{array}$ & 0,0018 \\
\hline $\mathrm{CT}$ & $\begin{array}{l}y=-0,313 x \\
8,164\end{array}$ & + & $\begin{array}{c}0,159 \\
3\end{array}$ & 0,0813 & $\begin{array}{l}y=-0,322 x \\
8,473\end{array}$ & $\begin{array}{c}0,236 \\
6\end{array}$ & 0,0296 & $\begin{array}{l}y=-0,757 x \\
8,5645\end{array}$ & $\begin{array}{c}0,467 \\
9\end{array}$ & 0,0009 \\
\hline EA & $\begin{array}{l}y=-2,633 x \\
52,20\end{array}$ & + & $\begin{array}{c}0,258 \\
9\end{array}$ & 0,0219 & $\begin{array}{l}y=-2,900 x \\
41,00\end{array}$ & $\begin{array}{c}0,215 \\
9\end{array}$ & 0,0390 & $\begin{array}{l}y=3,100 x \\
45,700\end{array}$ & $\begin{array}{c}0,257 \\
7\end{array}$ & 0,0223 \\
\hline G4 & $\begin{array}{l}y=-1,933 x \\
78,90\end{array}$ & + & $\begin{array}{c}0,093 \\
6\end{array}$ & 0,1897 & $\begin{array}{l}y=-3,700 x \\
86,00\end{array}$ & $\begin{array}{c}0,602 \\
8\end{array}$ & $<0,0001$ & $y=-1,067 x+68,20$ & $\begin{array}{c}0,020 \\
0\end{array}$ & 0,5519 \\
\hline G7 & $\begin{array}{l}y=-1,667 x \\
83,00\end{array}$ & + & $\begin{array}{c}0,068 \\
7\end{array}$ & 0,2643 & $\begin{array}{l}y=-3,833 x \\
88,30\end{array}$ & $\begin{array}{c}0,563 \\
4\end{array}$ & 0,0001 & $\begin{array}{l}y=-1,000 x \\
71,100\end{array}$ & $\begin{array}{c}0,020 \\
6\end{array}$ & 0,5462 \\
\hline $\begin{array}{c}\text { IV } \\
\text { E }\end{array}$ & $\begin{array}{l}y=\quad 0,076 x \\
2,211\end{array}$ & + & $\begin{array}{c}0,077 \\
2\end{array}$ & 0,2355 & $\begin{array}{l}y=-0,321 x \\
2,587\end{array}$ & $\begin{array}{c}0,817 \\
2\end{array}$ & $<0,0001$ & $y=-0,0045 x+2,733$ & $\begin{array}{c}0,000 \\
6\end{array}$ & 0,9193 \\
\hline
\end{tabular}




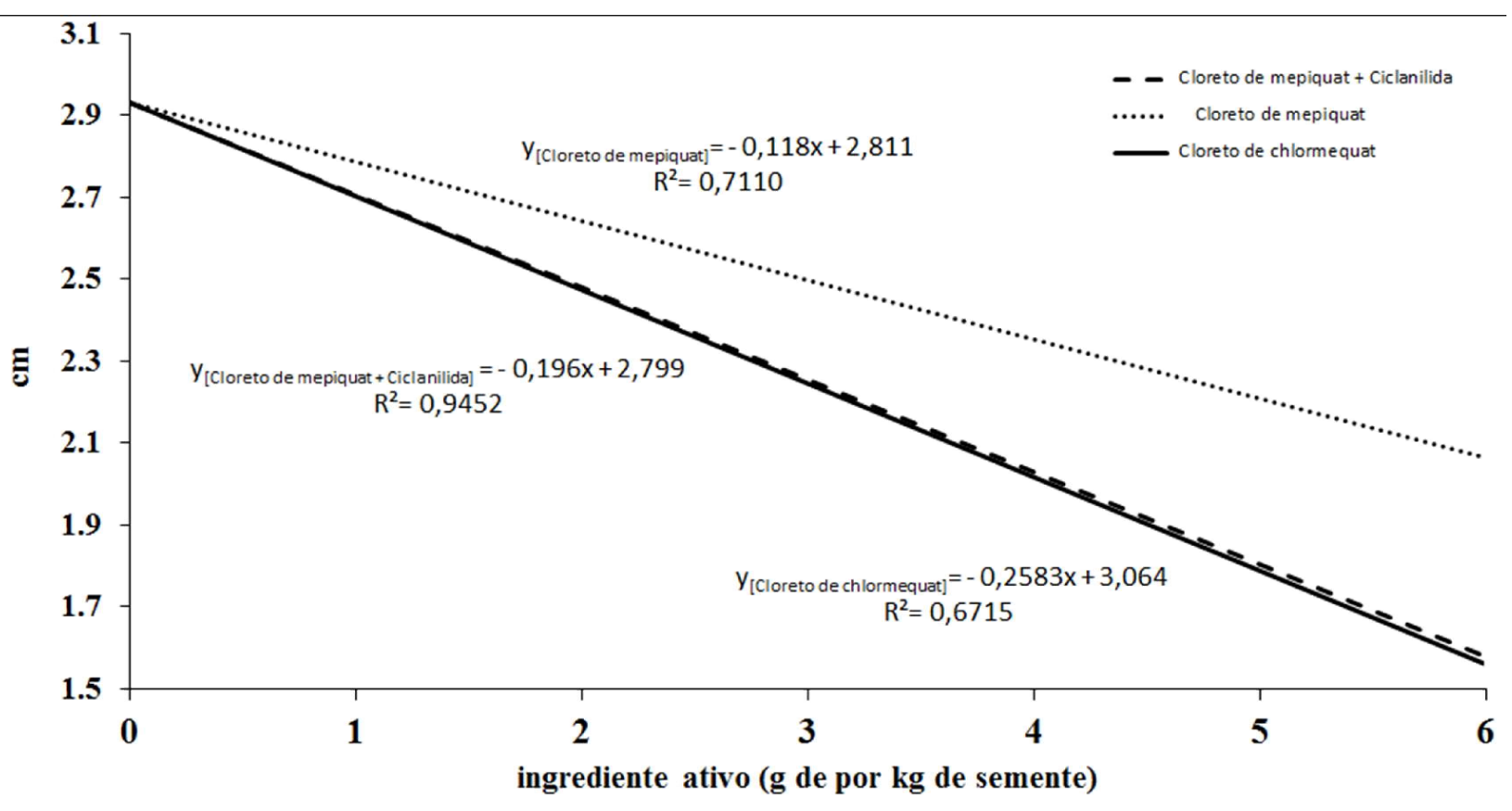

Figura 1. Análise de regressão para crescimento do hipocótilo $(\mathrm{cm})$ em função das doses dos reguladores de crescimento CM, CM+CY e CC, Piracicaba (SP), 2010.

Zhang, Cothren e Lorenz (1990), testando doses de até $2 \mathrm{~g}$ de cloreto de mepiquate por $\mathrm{kg}$ de sementes, observaram redução de massa de matéria seca de raiz. Porém, Nagashima et al. (2010a) concluiu que o comprimento da radícula das plântulas não foi reduzido significativamente em função das doses de cloreto de mepiquate utilizadas. Khan e Hayat (2005), e Soares (2010) também não observaram efeito do tratamento de sementes sobre crescimento radicular em profundidade.

Para comprimento total, houve redução do crescimento em função do aumento de doses de $\mathrm{CM}+\mathrm{CY}$ e $\mathrm{CC}$, sendo a redução do crescimento, para cada grama de ingrediente ativo por $\mathrm{kg}$ de semente aplicado, de 3,8 \% para $\mathrm{CM}+\mathrm{CY}$ e de $8,8 \%$ para CC (Tabela 4). Soares (2010) concluiu que o comprimento total das plântulas foi reduzido significativamente em função das doses de cloreto de mepiquate utilizadas, mencionando que o tratamento de sementes reduz a altura da planta já a partir da fase de plântula.

Comparando as médias do teste de envelhecimento acelerado, percebeu-se que o produto $\mathrm{CM}+\mathrm{CY}$ foi o único que reduziu a germinação de sementes, diferindo da testemunha e dos demais reguladores (Tabela 3). Todos os reguladores promoveram redução da germinação no teste de envelhecimento acelerado, sendo que a cada grama de ingrediente ativo por $\mathrm{kg}$ de 
semente ocorreu redução no crescimento de 7,0\% para $\mathrm{CM}+\mathrm{CY}$, de $6,8 \%$ para $\mathrm{CC}$ e, de 5,0\% para CM (Tabela 4$)$.

Os resultados de germinação aos quatro dias indicaram que todos os reguladores reduziram a germinação de sementes em relação à testemunha, retardando o processo de germinação, mas não houve diferença entre reguladores (Tabela 3). Já na contagem aos sete dias os reguladores que diferiram da testemunha, reduzindo a germinação foram $\mathrm{CM}+\mathrm{CY}$ e $\mathrm{CC}$; porém não houve diferença entre $\mathrm{CM}+\mathrm{CY}$ e $\mathrm{CM}, \mathrm{CM}+\mathrm{CY}$ e $\mathrm{CC}$, mas $\mathrm{CM}$ e $\mathrm{CC}$ diferiam.

Nagashima et al. (2010a) avaliando a qualidade fisiológica de sementes de algodão tratadas com cloreto de mepiquate nas doses: 2,5, 5 e 10 gi.a $\mathrm{kg}^{-1}$ de sementes e sem tratamento (testemunha) após diversos períodos de armazenamento, observaram que o percentual de germinação não diferiu entre os tratamentos, independentemente da dose utilizada.

Para contagem de germinação aos quatro e sete dias, o único produto que reduziu germinação em função do aumento de dose (Tabela 4) foi o produto $\mathrm{CM}+\mathrm{CY}$. A cada grama de ingrediente ativo por $\mathrm{kg}$ de semente houve $4,3 \%$ de redução na germinação aos quatro e sete dias.
Zhang, Cothren e Lorenz (1990) não verificaram efeito na germinação de sementes e emergência de plântulas ao testar até 2 gramas de cloreto de mepiquate por $\mathrm{kg}$ de semente. Pazzetti et al. (2009) também não verificaram efeito de cloreto de mepiquate e cloreto de clormequate na germinação e emergência de plântulas.

Para índice de velocidade de emergência, o único produto que diferiu da testemunha foi o $\mathrm{CM}+\mathrm{CY}$ (Tabela 3). Já os reguladores $\mathrm{CC}$ e $\mathrm{CM}$ não diferiram da testemunha, e diferiram do $\mathrm{CM}+\mathrm{CY}$, que também foi o único produto que promoveu redução no índice de velocidade de emergência em função do aumento de dose (Tabela 4). A cada grama de ingrediente ativo adicionado por $\mathrm{kg}$ de semente houve $12,4 \%$ de redução no índice de velocidade de emergência.

Soares (2010), em dois experimentos, verificou $16 \%$ e $5 \%$ de redução na emergência de plântulas para cada grama de cloreto de mepiquate associado à ciclanilidaaplicado por $\mathrm{kg}$ de sementes. Porém a diferença entre produtos foi contraditória nos experimentos. $\mathrm{O}$ autor ressaltou que houve uma tendência de redução da emergência de plântulas, sendo provavelmente o aumento da dose de cloreto de mepiquate, a associação da ciclanilida e o 
baixo vigor de sementes fatores que contribuem negativamente para a emergência.

Analisando os resultados, observouse que o tratamento de sementes com regulador de crescimento afetou a qualidade de sementes de algodoeiro, pois interferiu na condutividade elétrica, comprimento de plântula, comprimento de radícula, tendo todos os reguladores de crescimento afetado a germinação e a emergência de plântulas. No entanto, somente $\mathrm{CM}+\mathrm{CY}$ causou maior redução conforme o aumento de dose.

Também se verificou diferença entre o efeito dos reguladores testados, sendo os produtos mais efetivos $\mathrm{CM}+\mathrm{CY}, \mathrm{CC}$ e $\mathrm{CM}$, respectivamente. Analisando o efeito de aumento de dose dos reguladores observa-se ajuste de equação em $87,5 \%$ dos parâmetros analisados para $\mathrm{CM}+\mathrm{CY}, 50 \%$ para $\mathrm{CC}$, e $25 \%$ para CM (Tabela 4 ).

De maneira geral, o CM pareceu ser o ingrediente ativo mais apropriado ao tratamento de sementes de algodão, pois não afetou o crescimento de raiz como o produto $\mathrm{CC}$, e não interferiu na germinação de sementes e emergência de plântulas como o produto $\mathrm{CM}+\mathrm{CY}$, o qual demonstrou ser um produto mais efetivo, causando até efeitos indesejados, pois sempre promoveu resultados mais intensos que os demais produtos quando na mesma dose de ingrediente ativo.

\section{CONCLUSÕES}

De acordo com os resultados obtidos e a discussão, pode-se concluir que: (i) reguladores de crescimento aplicados via tratamento de sementes de algodoeiro interferem na qualidade fisiológica de sementes, afetando a germinação de sementes e emergência de plântulas; (ii) Há diferença entre o efeito dos reguladores de crescimento; (iii) Os produtos mais efetivos são:cloreto de mepiquate associado à ciclanilida, cloreto de clormequate e cloreto de mepiquate, respectivamente; e (iv) A associação àciclanilida intensifica o efeito de cloreto de mepiquate.

\section{REFERÊNCIAS BIBLIOGRÁFICAS}

BOX, G.E.; COX, D.R. 1964. Ananalysis of transformations. Journal of the Royal Statistical Society, London, v.26, p.211252.

BRASIL. 1992. Ministério da Agricultura e Reforma Agrária. Secretaria Nacional de Defesa Agropecuária. Departamento Nacional de Produção Vegetal. Coordenação de Laboratório Vegetal. Regras para Análise de Sementes. Brasília, DF, 365p.

CARVALHO, L.H.; CHIVEGATO, E.I.; KONDO, J.L.; SABINO, J.C.; PETTINELLI JUNIOR, A.; BORTOLETO, N.; GALLO, P.B. 1994. 
Fitorreguladores de crescimento e captação na cultura do algodoeiro. Bragantia, Campinas, v. 53, n. 2, p. 247-254.

CASTRO, P.R.C. 2006. Agroquímicos de controle hormonal na agricultura tropical. Série Produtor Rural, Piracicaba, n.32, p.46.

DUTRA, A.S.; MEDEIROS FILHO, S. 2009. Influência da pré-hidratação das sementes de algodão na resposta do teste de condutividade elétrica. Revista Brasileira de Oleaginosas Fibrosas. Campina Grande, v.13, n.2, p.45-52.

HARTLEY, H.O. 1950. The use of range in analysis of variance. Biometrika, London, v.37, p.271-280.

JOST, P.H.; COTHREN, J.T. 2001. Phenotypic alterations and crop maturity differences in ultra-narrow row and conventionally spaced cotton. Crop Science, Amsterdam, v.41, p.1150-1159

KHAN, M.I.N.N.; HAYAT, R.S.A.K. 2005. Contribution of mepiquate chloride in drought tolerance in cotton seedlings. Asian Journal of Plant Sciences, Faisalabad, v.4, n.5, p.530-532.

LACA-BUENDIA, J.P. 1989. Efeito de reguladores de crescimento no algodoeiro (Gossypium hirsitum L.) Revista Brasileira de Fisiologia Vegetal. Brasília, v.1, n.1, p. 109-113.

LAMAS, F.M. 2001. Reguladores de Crescimento. In: Embrapa Agropecuária Oeste. Algodão: tecnologia de produção. Embrapa Agropecuária Oeste; Embrapa Algodão, Dourados, 296p.

LAMAS, F.M. 2006. Fitorreguladores bem manejados equilibram crescimento da planta. Visão Agrícola, Piracicaba, n.6, p.81-84.

MAGUIRE, J.D. 1962. Speed of germination, aid in selection and evolution for seedling emergence and vigor. Crop Science. Madson, v.2, n.2, p.176-177.
MARCOS FILHO, J.; CÍCERO, S.M.; SILVA, W.R. 1987. Avaliação da qualidade das sementes. Piracicaba: FEALQ, 230 p.

MARUR, C.J. 1998. Fotossíntese e translocação de carboidratos em algodoeiros submetidos a déficit hídrico após aplicação de cloreto de mepiquate. Revista Brasileira de Fisiologia. Brasília, v.10, n.1, p.59-64.

MARUR, C.J.; RUANO, O. 2001. A reference system for determinationofdevelopmentalstagesofup landcotton. Revista de Oleaginosas e Fibrosas, Campina Grande, v.5, p.313317.

McCARTY, J.C.; HEDIN, P.A. 1994. Effects of 1,1-dimethylpiperidinium chloride on the yields, agronomic traits, and allelochemicals of cotton (Gossypium hirsutum L.), a nine years study. Journal of Agricultural and Food Chemistry, Easton, v.42, p.23022304.

NAGASHIMA， G.T.; MARUR， C.J.; YAMAOKA, R.S.; MIGLIORANZA, E. 2005. Desenvolvimento inicial de plantas de algodão provenientes de sementes embebidas em cloreto de mepiquate. Pesquisa Agropecuária Brasileira, Brasília, Notas científicas.

NAGASHIMA, G.T.; MIGLIORANZA, E.; MARUR, C.J.; YAMAOKA, R.S.; GOMES, J.C. 2007.Embebição de sementes e aplicação foliar com cloreto de mepiquate no crescimento e produção do algodoeiro. Ciência e Agrotecnologia, Lavras, v.31, n.4, p.1027-1034.

NAGASHIMA, G.T.; MIGLIORANZA, E.; MARUR, C.J.; YAMAOKA, R.S.; BARROS A.S.R.; MARCHIOTTO, F. 2010a. Qualidade fisiológica e armazenamento de sementes de algodão embebidas em solução de cloreto de mepiquate. Ciência Agrotecnica. Lavras, v. 34, n. 3, p. 681-687. 
NAGASHIMA, G.T.; MIGLIORANZA, E.; MARUR, C.J.; YAMAOKA, R.S.; SILVA, J.G.R. 2010b. Desenvolvimento inicial de plantas de algodão provenientes de sementes embebidas em cloreto de mepiquate. Ciência Rural, Santa Maria, v.40, n.1.

NICHOLS, S.P.; SNIPES, C.E.; JONES, M.A. 2003. Evaluation of row spacing and mepiquate chloride in cotton. Journal of Cotton Science, v.7, p.148155.

PAZZETTI G.A.; NASCIMENTO, A.; SCHWENING, F.F.; CARVALHO, C.L. 2009.Gerenciamento de crescimento pela aplicação de regulador de crescimento via semente e via foliar. CONGRESSO BRASILEIRO DO ALGODÃO, 7, Foz do Iguaçu. Sustentabilidade da cotonicultura Brasileira e Expansão dos Mercados: Anais... Campina Grande: Embrapa Algodão, p. 820-828.

PEREIRA J.R.; ARAUJO W.P.; JÚNIOR. G.S.C.; LIMA, F.V. de.; JUNIOR, S.P.S. 2008. Modos de aplicação do Cloreto de Mepiquate em Duas Cultivares de Algodoeiro Herbáceo. Campina Grande, PB, Embrapa Algodão. 7 p.

REDDY, K.R.; BOONE, M.L.; REDDY, A.R.; HODGES, H.F.; TURNER, S.B.; McKINION, J.M. 1995. Developing and validating a model for plant growth regulator. Agronomy Journal, Madison, v. 87 , n. 6 , p. 1100-1105.

SALISBURY, F.B.; ROSS, C.W. 1991.Plant physiology. Belmont: Wadsworth, 682p.

SOARES, L.C.S. 2010. Efeito de reguladores de crescimento (via tratamento de sementes e foliar) no desenvolvimento e na produtividade da cultura de algodão. 2010. 131p. Dissertação (Mestrado em Fitotecnia) Escola Superior de Agricultura "Luiz de Queiroz", Universidade de São Paulo, Piracicaba.
SWAIN, S.M.; SINGH, D.P. 2005. Tall tales from sly dwarves: movel functions of gibberellins in plant development. Trend Plants Science, Londres, v.10, n.3, p. 123-129.

TAIZ, L.; ZEIGER, E. 2004.Fisiologia vegetal. Porto Alegre: Artmed. p.570554.

VIEIRA, R.D., KRZYZANOWSKI, F.C. 1999. Teste de condutividade elétrica. In: KRZYZANOWSKI, F.C., VIEIRA, R.D., FRANÇA NETO, J.B. (Ed.). Vigor de sementes: conceitos e testes. Londrina: ABRATES, p.4.1-4.26.

YAMAOKA, R.S.; ALMEIDA, W.P. de; PIRES, J.R.; MARUR, C.J.; NAGASHIMA, G.T.; SILVA, A.V. 2001. Comportamento de cultivares IPR 95 e Coodetec 401 ao adensamento de plantio do algodoeiro no estado do Paraná. In: CONGRESSO BRASILEIRO DE ALGODÃO, 3., Campo Grande. Resumos... Campo Grande: UFMS; Campina Grande: EMBRAPA-CNPA; Dourados: EMBRAPA-CPAO, v.1. p.609-611.

ZHANG, S.; COTHREN, J.T.; LORENZ, E.J. 1990.Mepiquate chloride seed treatment and germination temperature effects on cotton growth, nutrient partition, and water use efficiency. Journal of Plant Growth Regulation, New York, v.9, p. 195-199.

Recebido em: 10/4/2015 Aceito para publicação em: 22/9/2015 